\title{
Aporte e decomposição de serapilheira em Floresta Estacional Decidual na região do Alto Uruguai, $R S$
}

\author{
Felipe Turchetto ${ }^{1}$, Fabiano de Oliveira Fortes ${ }^{2}$ \\ ${ }^{1}$ Universidade Federal de Santa Maria, Departamento de Ciências Florestais, Campus Universitário, CP 281, Camobi, CEP 97.110-970, Santa Maria, RS, Brasil \\ ${ }^{2}$ Universidade Federal de Santa Maria, Departamento de Engenharia Florestal, Campus Frederico Westphalen, CP 54, Linha 7 de Setembro, CEP 98.400-000, \\ Frederico Westphalen, RS, Brasil
}

"Autor correspondente:
turchetto.felipe@gmail.com

Termos para indexação:

Mineralização

Sazonalidade

Decomposição

Index terms:

Mineralization

Seasonality

Decomposition

Histórico do artigo:

Recebido em 18/06/2014

Aprovado em 12/12/2014

Publicado em 31/12/2014

doi: 10.4336/2014.pfb.34.80.735
Resumo - O presente estudo teve por objetivo analisar e descrever a dinâmica sazonal da deposição e decomposição de serapilheira na Floresta Estacional Decidual da região do Médio Alto Uruguai, RS. Para a determinação da deposição de serapilheira foram instalados 20 coletores de $1 \mathrm{~m}^{2}$ em uma área de um hectare, sendo realizadas avaliações mensais. Para a amostra da serapilheira acumulada nas estações sazonais, foram coletadas 20 amostras aleatórias em molduras de $25 \mathrm{~cm} \mathrm{x} 25 \mathrm{~cm}$. O material coletado foi identificado e separado nas frações: folhas, galhos e miscelânea. Após a estratificação, as amostras foram secas em estufa a $70^{\circ} \mathrm{C}$ e pesadas. A deposição anual de serapilheira foi estimada em 7,14 t.ha-1, sendo composta por $58,54 \%$ de folhas, $28,69 \%$ de galhos e $12,77 \%$ de miscelânea. A deposição de serapilheira total foi contínua ao longo do período de estudo, apresentando modelo sazonal, com os maiores valores no final do inverno e início da primavera. O estoque médio de serapilheira foi estimado em 6,50 t.ha $^{-1}$. A taxa de decomposição foi de 1,097, constatando-se a rápida mineralização da serapilheira produzida.

\section{Litter deposition and decomposition in Deciduous Forest in the Upper Uruguay region, $R S$}

\begin{abstract}
The present study aimed to analyze and describe the seasonal dynamics of deposition and decomposition of litter in the Deciduous Forest in the Middle Upper Uruguay region, Rio Grande do Sul State, Brazil. To determine the deposition of litter, 20 collectors of $1 \mathrm{~m}^{2}$ were installed in an area of one hectare, with evaluations conducted monthly. For litter accumulated in seasonal stations, 20 random samples were collected in frames of $25 \mathrm{~cm} \times 25 \mathrm{~cm}$. The collected material was identified and separated into fractions of leaves, small twigs and miscellaneous. After stratification, the samples were dried at $70{ }^{\circ} \mathrm{C}$ and weighted. The estimated annual litter production was $7.14 \mathrm{t} \mathrm{ha}^{-1}$, consisting of $58.54 \%$ of leaves, $28.69 \%$ of small twigs and $12.77 \%$ for miscellaneous. The deposition of litter was continuous throughout the studying period, showing a seasonal pattern, with higher values in late winter and early spring. The estimated average stock of litter was $6.50 \mathrm{t} \mathrm{ha}^{-1}$. The decomposition rate was 1.097 , showing a fast litter mineralization.
\end{abstract}




\section{Introdução}

A serapilheira acumulada sobre o solo das florestas tem um importante papel na dinâmica dos ecossistemas. $\mathrm{O}$ aporte de serapilheira pode variar de acordo com o estádio sucessional da formação florestal (Delitti, 1989), o grau de perturbação da área (Martins \& Rodrigues, 1999) e o tipo de vegetação (Moreira \& Silva, 2004).

O processo de decomposição da serapilheira mantém a funcionalidade do ecossistema (Olson, 1963), constituindo o maior caminho biológico na transferência de nutrientes e na regulação dos teores de matéria orgânica do solo (Xu \& Hirata, 2002; Schumacher et al., 2004).

A quantificação do aporte e decomposição de serapilheira tem contribuído para a compreensão do funcionamento e manejo de florestas, particularmente nas regiões tropicais (Jordan, 1985; Delitti, 1995). O conhecimento dos processos relacionados ao aporte e a transformação da serapilheira tem sido considerado um valioso instrumento para estudos de diagnóstico ambiental e da intensidade dos impactos naturais ou antrópicos (Cianciaruso et al., 2006).

Nesse contexto, torna-se fundamental o conhecimento das variações de aporte e decomposição de serapilheira em ecossistemas naturais, pois permitem obter um conjunto de informações que podem contribuir para um melhor entendimento da dinâmica de coberturas florestais nativas, tornando-se também uma ferramenta útil para o manejo e conservação destas florestas. Nesse sentido, o objetivo deste estudo foi quantificar a deposição, o aporte e o tempo de renovação do estoque de serapilheira em um fragmento de Floresta Estacional Decidual, na região do Médio Alto Uruguai, no Estado do Rio Grande do Sul.

\section{Material e métodos}

O fragmento estudado possui aproximadamente 20 ha de Floresta Estacional Decidual. Está localizado no Município de Taquaruçu do Sul, RS, sob as coordenadas geográficas $53^{\circ} 25^{\prime} 57,54^{\prime \prime} \mathrm{O}$ e $27^{\circ} 23^{\prime} 55,98^{\prime \prime} \mathrm{S}$, com altitude variando entre $520 \mathrm{~m}$ a $550 \mathrm{~m}$.

Os dados climáticos (temperatura, umidade, precipitação e velocidade do vento) foram obtidos na estação meteorológica localizada no Centro de Educação Superior Norte do RS, pertencente à Universidade Federal de Santa Maria (UFSM). O clima da região de estudo, de acordo com a classificação climática de
Köppen é subtropical úmido (Alvares et al., 2013), com temperatura média anual de $19{ }^{\circ} \mathrm{C}$, com máxima de $38{ }^{\circ} \mathrm{C}$ e mínima de $0{ }^{\circ} \mathrm{C}$. Os solos do Município de Taquaruçu do Sul derivam da decomposição de rochas basálticas, sendo predominantemente classificados como Latossolos Vermelhos Aluminoférricos típicos (Cunha et al., 2010).

Para a coleta da serapilheira depositada, foram distribuídos de forma aleatória 20 coletores de $1 \mathrm{~m}^{2}(1 \mathrm{~m}$ x $1 \mathrm{~m}$ ), em uma área de um hectare, que foram fixados a aproximadamente $10 \mathrm{~cm}$ acima da superfície do terreno.

As coletas de serapilheira foram realizadas mensalmente, durante um período de um ano (de julho de 2010 a junho de 2011). O material coletado foi devidamente identificado e levado ao Laboratório de Silvicultura, do Departamento de Engenharia Florestal, da UFSM, onde foram separadas as frações: folhas, galhos e miscelânea (flores, frutos, sementes e restos de vegetais não identificados). Após a estratificação, as amostras foram secas em estufa a $70{ }^{\circ} \mathrm{C}$, sendo efetuada a pesagem em balança de precisão, obtendo-se assim o peso de cada fração.

Para determinação do estoque de serapilheira no solo foram realizadas avaliações sazonais nas quatro estações do ano (primavera, verão, outono e inverno). Foram coletadas 20 amostras de serapilheira em quadrantes de $25 \mathrm{~cm} \times 25 \mathrm{~cm}$ (Fortes, 2007), nas proximidades de cada caixa de coleta de serapilheira depositada. O material coletado foi peneirado em tela de malha de 2,0 $\mathrm{mm}$ e separado em folhas, galhos e miscelânea. Posteriormente, o material foi seco em estufa e pesado.

O estoque médio de serapilheira acumulada por hectare sobre o solo foi calculado segundo a equação (Lopes et al., 2002):

$$
E S=(P S \times 10.000) / A g
$$

Em que: $\mathrm{ES}=$ estoque de serapilheira $\left(\mathrm{kg} \cdot \mathrm{ha}^{-1}\right)$; $\mathrm{PS}=$ peso seco médio $(\mathrm{kg})$ e $\mathrm{Ag}=$ área do gabarito $\left(\mathrm{m}^{2}\right)$.

A taxa de decomposição da serapilheira foi estimada por meio da equação proposta por Olson (1963), e empregada em estudos semelhantes (Kolm \& Poggiani, 2003; Arato et al., 2003):

$K=L / X_{s s}$

Em que: $K=$ constante de decomposição; $L=$ deposição anual de serapilheira $\left(\mathrm{g} \cdot \mathrm{m}^{-2}\right)$ e $X_{s s}=$ média anual da serapilheira acumulada sobre o solo $\left(\mathrm{g} \cdot \mathrm{m}^{-2}\right)$.

A taxa de decomposição instantânea é a relação entre a massa de serapilheira depositada e a massa de serapilheira acumulada. A partir do valor de K, calculou-se, também, 
o tempo médio de renovação estimado por $1 / \mathrm{K}$ e os tempos necessários para que ocorra decomposição de $50 \%$ (t 0,5) e 95\% (t 0,05) da serapilheira, estimados pela equação de Shanks \& Olson (1961):

$$
t 0,5=\ln \frac{2}{k} \quad t 0,5=\frac{3}{k}
$$

Os resultados foram submetidos ao Teste de Normalidade de Shapiro-Wilk e ao Teste de Homogeneidade de Variâncias de Bartlett. Em seguida, realizou-se a análise de variância e a comparação de médias pelo teste de Scott-Knott, $p<0,05$, utilizando o software SISVAR v. 5.3 (Ferreira, 2008). A correlação entre dados climáticos (precipitação, temperatura média e máxima, velocidade do vento, umidade relativa do ar) e de produção e acúmulo da serapilheira foi analisada por intermédio da matriz de correlação de Pearson.

\section{Resultados e discussão}

A deposição total de serapilheira ao longo do período

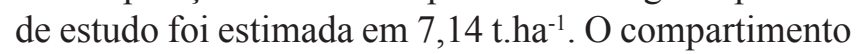
folhas correspondeu a maior porção (58,54\%), com deposição anual de 4,15 t.ha ${ }^{-1}$. A fração galhos apresentou produção anual de 2,07 t.ha' ${ }^{-1}$, perfazendo
28,69\% do total depositado e a fração miscelânea ficou com $12,77 \%$ do total $\left(0,92\right.$ t.ha $\left.^{-1}\right)$ (Tabela 1$)$.

Como observado por alguns autores (König et al., 2002; Bonacina et al., 2005) a serapilheira é formada em sua maioria por folhas (60 a 70\%), sendo a miscelânea a menor fração encontrada . Tal padrão de distribuição pode estar relacionado ao estado fisiológico em que a planta se encontra, seja pelo período de repouso vegetativo ou como resposta ao estresse, a qual é submetida em virtude de variações climáticas. A folha é responsável pelas trocas gasosas da planta com o ambiente, em situações de estresse. Uma estratégia desenvolvida para suportar esse fenômeno é a redução da área foliar, consequentemente uma quantidade maior deste compartimento será evidenciada na constituição da serapilheira.

$\mathrm{Na}$ análise da distribuição temporal, observou-se diferença significativa $(\mathrm{p}<0,05)$, sendo nos meses de julho e setembro os picos de deposição, corroborando König et al. (2002), que em estudo de deposição de serapilheira em Floresta Estacional Decidual próxima ao Município de Santa Maria, RS, verificaram os maiores picos de deposição de serapilheira entre os meses de julho a setembro.

Tabela 1. Variação sazonal da deposição das diferentes frações (folhas, galhos e miscelânea) e da serapilheira total, em fragmento de Floresta Estacional Decidual, Taquaruçu do Sul, RS.

\begin{tabular}{lcccc}
\hline \multirow{2}{*}{ Fator } & \multicolumn{4}{c}{ Compartimento $\left(\right.$ Kg ha $\left.^{-1}\right)$} \\
\cline { 2 - 5 } & Folhas & Galhos & Miscelânea & Serapilheira \\
\hline Julho & $519,3 \mathrm{~A}$ & $303,2 \mathrm{~A}$ & $111,7 \mathrm{~B}$ & $934,2 \mathrm{~A}$ \\
Agosto & $357,9 \mathrm{~B}$ & $181,5 \mathrm{~B}$ & $84,5 \mathrm{~B}$ & $623,9 \mathrm{~B}$ \\
Setembro & $591,9 \mathrm{~A}$ & $348,2 \mathrm{~A}$ & $148,5 \mathrm{~A}$ & $1088,7 \mathrm{~A}$ \\
Outubro & $389,5 \mathrm{~B}$ & $192,1 \mathrm{~B}$ & $101,5 \mathrm{~B}$ & $683,1 \mathrm{~B}$ \\
Novembro & $302,1 \mathrm{~B}$ & $133,2 \mathrm{~B}$ & $98,0 \mathrm{~B}$ & $533,2 \mathrm{C}$ \\
Dezembro & $208,8 \mathrm{C}$ & $17,3,8 \mathrm{~B}$ & $89,1 \mathrm{~B}$ & $471,7 \mathrm{C}$ \\
Janeiro & $233,9 \mathrm{C}$ & $154,3 \mathrm{~B}$ & $87,9 \mathrm{~B}$ & $476,2 \mathrm{C}$ \\
Fevereiro & $263,3 \mathrm{C}$ & $83,9 \mathrm{C}$ & $49,0 \mathrm{C}$ & $396,3 \mathrm{C}$ \\
Março & $369,5 \mathrm{~B}$ & $98,6 \mathrm{C}$ & $31,9 \mathrm{C}$ & $500,1 \mathrm{C}$ \\
Abril & $312,8 \mathrm{~B}$ & $243,2 \mathrm{~B}$ & $66,7 \mathrm{C}$ & $622,7 \mathrm{~B}$ \\
Maio & $227,8 \mathrm{C}$ & $56 \mathrm{C}$ & $29,4 \mathrm{C}$ & $313,2 \mathrm{C}$ \\
Junho & $372,7 \mathrm{~B}$ & $102,1 \mathrm{C}$ & $20,3 \mathrm{C}$ & $495,1 \mathrm{C}$ \\
\hline Total (Kg.ha.ano $\left.{ }^{-1}\right)$ & 4149,68 & 2070,28 & 918,57 & 7138,53 \\
$F(p)$ & $14,997(0,000)$ & $7,464(0,000)$ & $9,505(0,000)$ & $15,121(0,000)$ \\
CV (\%) & 13,45 & 45,67 & 21,76 & 15,98 \\
\hline
\end{tabular}

Em que: $F=$ estatística do teste Snedecor; $P=$ probabilidade estatística. Médias seguidas de mesma letra maiúscula na linha não diferem a $5 \%$ pelo teste de Scott-Knott. 
O período em que as plantas apresentam o seu máximo vigor de crescimento fica entre os meses de outubro/ novembro (início da primavera) a abril/maio (início do inverno) . Desse modo, a folhagem permanece intacta a não ser por ocorrência de fenômenos naturais, justificando assim, os menores valores encontrados para o período.

Os dados da Tabela 2 demostram que não há correlação entre a deposição das diferentes frações de serapilheira com as variáveis climáticas (precipitação, vento, temperatura e umidade). König et al. (2002), em Floresta Estacional Decidual próxima ao Município de Santa Maria, RS, verificaram que a deposição de serapilheira apresentou correlações negativas com a temperatura, não sendo significativa com a precipitação. No entanto, Figueiredo Filho et al. (2003) obtiveram baixa correlação linear com a precipitação $(r=0,36)$ e com a temperatura $(\mathrm{r}=-0,10)$, em uma Floresta Ombrófila Mista no sul do Paraná.

Observa-se um determinado padrão de sazonalidade da deposição de serapilheira, com picos de deposição nos meses de julho e setembro (Figura 1). Esse padrão de deposição é típico de florestas estacionais (Arato et al., 2003), com pico de deposição no final do inverno/ início da primavera, como resposta a estacionalidade climática (Schlittler et al., 1993; Martins \& Rodrigues, 1999; Werneck et al., 2001), ocorrendo a estagnação do crescimento na estação mais fria, que se caracteriza como uma estratégia das espécies presentes nesta formação. Nesse período a folhagem senescente é eliminada e depositada sobre o solo, visando o aparecimento de novas folhas no período de crescimento que se inicia com a primavera.

\section{Serapilheira acumulada}

Tanto para os estratos, quanto para o total de serapilheira acumulada, observaram-se diferenças significativas entre as quatro estações (Figura 2). A quantidade de serapilheira acumulada sobre o solo seguiu padrão sazonal de distribuição, tanto nos estratos quanto para o total, ratificando relatos observados em outros trabalhos (Borém \& Ramos, 2002; König et al., 2002; Figueiredo Filho et al., 2003; Moreira \& Silva, 2004; Fortes, 2007).

$\mathrm{O}$ estoque médio de serapilheira acumulado sobre o solo foi estimado em 6,50 t.ha- ${ }^{-1}$. Constatou-se que o inverno apresentou a maior média entre as estações sazonais, com 8,97 tha ${ }^{-1}$. A menor média foi encontrada no verão $\left(4,83\right.$ t.ha $\left.^{-1}\right)$, o que demonstra grande variação sazonal dentro da mesma tipologia florestal.

Com as baixas temperaturas e uma menor radiação solar no inverno, ocorre uma redução e ou estagnação da atividade microbiológica, consequentemente, ocorre uma menor ciclagem do material depositado na floresta. Portanto, nessa estação o acumulo de serapilheira no solo torna-se maior que a decomposição, ocasionando uma maior quantidade de material encontrado.

Em função da quantidade elevada de material acumulado no solo da floresta e com a menor decomposição no inverno, na estação subsequente (primavera), em que as plantas iniciam as brotações e floração, obteve-se um grande acúmulo de serapilheira $\left(7,24\right.$ t.ha $\left.^{-1}\right)$. Isso se deve à existência de material acumulado na estação anterior (inverno), que ainda não foi decomposto, embora na primavera aumente a decomposição em relação à deposição.

Tabela 2. Índice de correlação de Pearson, entre os componentes da serapilheira e as variáveis ambientais, em Floresta Estacional Decidual, Taquaruçu do Sul, RS.

\begin{tabular}{|c|c|c|c|c|c|c|c|c|}
\hline & Folhas & Galhos & Miscelânea & Serapilheira & Precipitação & Temperatura & Umidade & Vento \\
\hline Folhas & 1 & $0,750 *$ & $0,544^{\mathrm{ns}}$ & $0,921 *$ & $0,303^{\mathrm{ns}}$ & $-0,436^{\mathrm{ns}}$ & $-0,161^{\mathrm{ns}}$ & $0,271^{\mathrm{ns}}$ \\
\hline Galhos & & 1 & $0,835^{*}$ & $0,940 *$ & $0,334^{\mathrm{ns}}$ & $-0,132^{\text {ns }}$ & $-0,221^{\mathrm{ns}}$ & $-0,062^{\text {ns }}$ \\
\hline Miscelânea & & & 1 & $0,794 *$ & $0,166^{\mathrm{ns}}$ & $0,106^{\mathrm{ns}}$ & $-0,525^{\mathrm{ns}}$ & $0,089^{\text {ns }}$ \\
\hline Serapilheira & & & & 1 & $0,322^{\mathrm{ns}}$ & $-0,264^{\mathrm{ns}}$ & $-0,260^{\mathrm{ns}}$ & $0,135^{\mathrm{ns}}$ \\
\hline Precipitação & & & & & 1 & $0,277^{\text {ns }}$ & $0,488^{\text {ns }}$ & $0,176^{\mathrm{ns}}$ \\
\hline Temperatura & & & & & & 1 & $-0,092^{\mathrm{ns}}$ & $-0,347^{\text {ns }}$ \\
\hline Umidade & & & & & & & 1 & $-0,094^{\mathrm{ns}}$ \\
\hline Vento & & & & & & & & 1 \\
\hline
\end{tabular}

Em que: * Significativo a nível de $5 \%$ de probabilidade pelo teste $\mathrm{F}$; ${ }^{\text {ns }}$ não significativo 

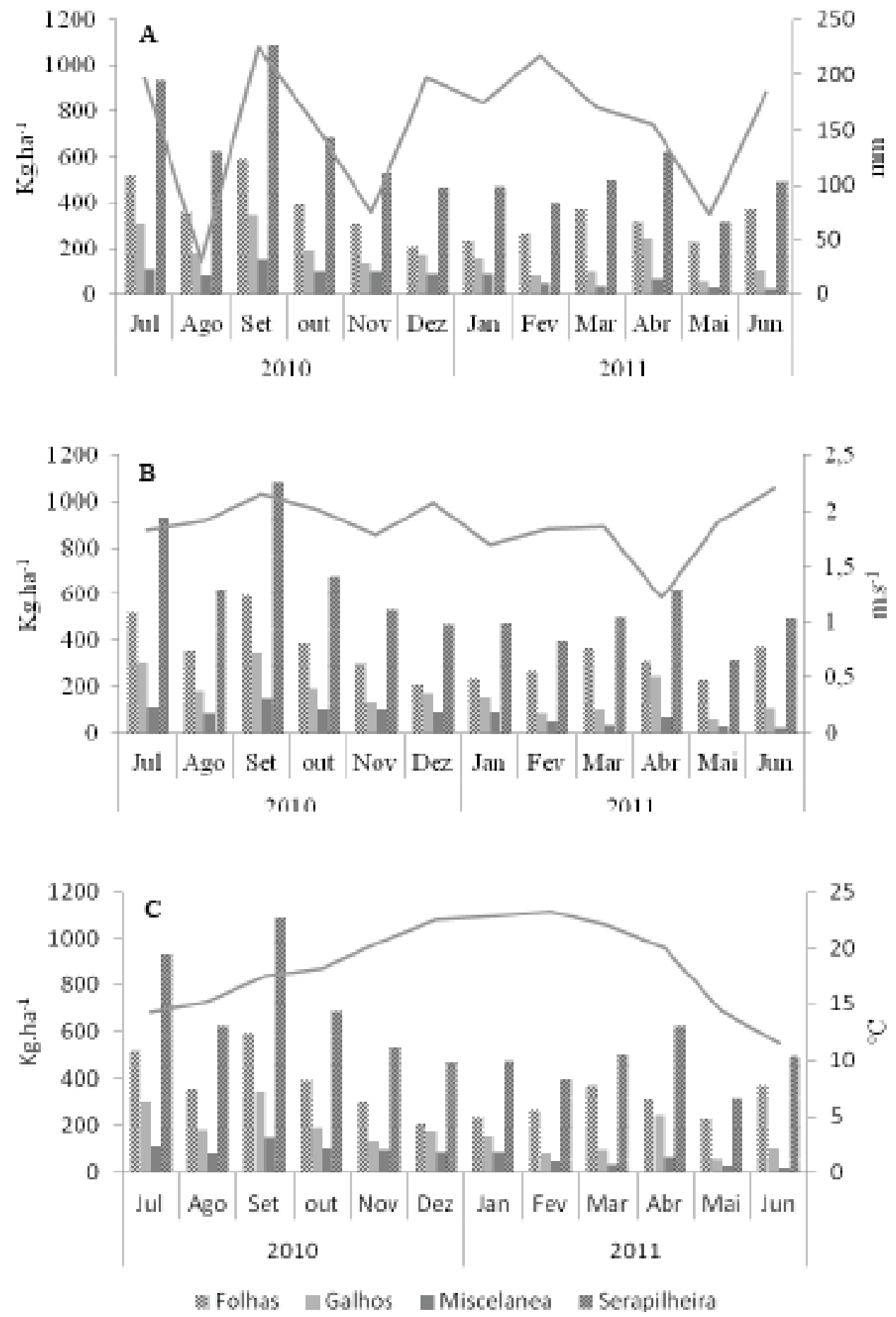

Figura 1. Variação mensal da deposição de serapilheira $\left(\mathrm{kg} \cdot \mathrm{ha}^{-1}\right)$ : A) Com a pluviosidade; B) Velocidade do vento e C) Temperatura, em Floresta Estacional Decidual, Taquaruçu do Sul, RS. 


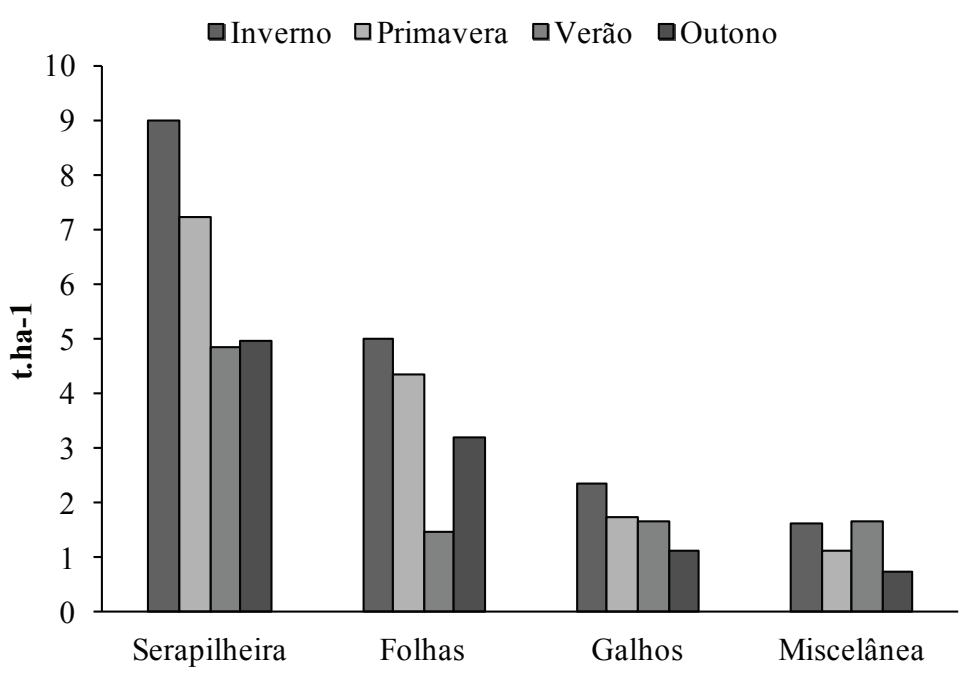

Figura 2. Sazonalidade do acumulo de serapilheira (folhas, galhos e miscelânea) e das diferentes frações. Médias seguidas de uma mesma letra para o mesmo compartimento de serapilheira, não diferem entre si, pelo teste de Scott-Knott a 5\% de probabilidade.

Nas estações sazonais verão e outono, encontraram-se as menores médias de serapilheira, tendência também revelada por Cunha et al. (1993) em fragmentos de Floresta Estacional Decidual e por Fortes (2007) estudando um fragmento de Floresta Ombrófila Mista. A menor quantidade de material depositado, em virtude da estacionalidade climática, bem como as maiores taxas de transformação de serapilheira, podem ser considerados como responsáveis para o menor acúmulo de serapilheira sobre o solo de florestas tropicais nos períodos de novembro a março.

\section{Decomposição da serapilheira}

A taxa de decomposição da serapilheira $(\mathrm{K})$ foi de 1,097 , próximo aos encontrados em florestas estacionais semideciduais, com valores de 1,02 (Schilittler et al., 1993) a 1,6 ((Morellato, 1992). Para um fragmento de Floresta Estacional Decidual, como o aqui estudado, Cunha et al. (1993) encontrou K igual a 1,2. Diferenças na taxa de decomposição da serapilheira podem estar relacionados a fatores como a fauna do solo e as condições ambientais, especialmente temperatura e a umidade (Anderson et al., 1983; César, 1993), os quais atuam como limitantes da atividade microbiológica,

O tempo de renovação do estoque foi inferior a um ano $(0,911)$, valor semelhante ao encontrado por Vital et al. (2004) estudando a Floresta Estacional Semidecidual em Zona Ripária. O tempo necessário para o desaparecimento de $50 \%$ e $95 \%$ da serapilheira foi, respectivamente, de 228 e 983 dias, valores próximos aos encontrados por Cunha et al. (1993) para o mesmo tipo de fitofisionomia (216 e 933 dias, respectivamente).

A rápida transformação da serapilheira, depositada ao longo do ano, está relacionada às elevadas temperaturas ocorrentes (Figura 1C), favorecendo a ação dos microorganismos responsáveis pela decomposição da matéria orgânica. Parte desse material passa pelo processo de humificação e permanece no solo como húmus estável. O restante deste material passa pelo processo de mineralização, no qual a matéria orgânica é transformada em compostos simples, solúveis ou gasosos. Uma vez disponíveis, esses compostos desempenham papel fundamental nos processos ecossistêmicos, servindo de base para os processos biogeoquímicos, que são responsáveis pela estabilidade ecossistêmica.

\section{Conclusões}

A deposição de serapilheira apresentou padrão sazonal de deposição, com pico de deposição no mês de setembro, respondendo a estacionalidade climática, porém não havendo correlação entre a serapilheira depositada e as variáveis climáticas (temperatura, pluviosidade e velocidade do vento).

A quantidade de serapilheira acumulada é diferente nas quatro estações, apresentando sazonalidade ao longo do ano.

A taxa de decomposição foi de 1,09, o que indica um rápido retorno (disponibilização) dos nutrientes ciclados para o ecossistema local. 


\section{Referências}

ALVARES, C. A.; STAPE, J. L.; SENTELHAS, P. C.; GONÇALVES, J. L. M.; SPAROVEK G. Köppen's climate classification map for Brazil. Meteorologische Zeitschrift, Berlin, v. 22, n. 6, p. 711-728, 2013.

ANDERSON, J. M.; PROCTOR, J.; VALLACK, H. W. Ecological studies in four constrasting lowland rain forest in Gunung Mulu National Park, Sarawak. III. Decomposition processes and nutrient losses from leaf litter. Journal of Ecology, v. 71, n. 3, p. 503-527, 1983.

ARATO, H. D.; MARTINS, S. V.; FERRARI, S. H. S. Produção e decomposição de serapilheira em um sistema agroflorestal implantado para recuperação de área degradada em Viçosa- MG. Revista Árvore, Viçosa, MG, v. 27, n. 5, p. 715-721, 2003.

BONACINA, D. M.; BRUN, E. J.; SCHUMACHER, M. V.; VIERA, M. Deposição de serapilheira em três estágios sucessionais de uma floresta estacional decidual em Santa Tereza, RS. In: CONGRESSO BRASILEIRO DE CIÊNCIA DO SOLO, 30., 2005, Recife. Solos: sustentabilidade e qualidade ambiental: anais... Recife: Sociedade Brasileira de Ciência do Solo, 2005.

BORÉM, R. A. T.; RAMOS, D. P. Variação estacional e topográfica de nutrientes na serapilheira de um fragmento de Mata Atlântica. Revista Cerne, Lavras, n. 2, p. 42-59, 2002.

CÉSAR, O. Produção de serapilheira na mata mesófila semidecídua da Fazenda Barreiro Rico, município de Anhembi, SP. Revista Brasileira de Biologia, Rio de Janeiro, v. 53, n. 4, p. 671-681, 1993.

CIANCIARUSO, M. C.; PIRES, J. S. R.; DELITTI, W. B. C.; SILVA, E. F. L. P. Produção de serapilheira e decomposição do material foliar em um Cerradão na Estação Ecológica de Jataí, município de Luiz Antônio, SP, Brasil. Acta Botânica Brasílica, São Paulo, v. 20, n. 1, p. 49-59, 2006.

CUNHA, G. C. GRENDENE, L. A.; DURLO, M. A.; BRESSAN, D. A. Dinâmica nutricional em Floresta Estacional Decidual com ênfase aos minerais provenientes da deposição da serapilheira. Ciência Florestal, Santa Maria, RS, n. 1, p. 35-64, 1993.

CUNHA, N. G.; SILVEIRA, R. J. C.; KOESTER, E.; OLIVEIRA, L. D.; ALBA, J. M. F.; COSTA, F. A.; TERRES, V. C.; LOPES, R. T. Estudos de solos do município de Taquaruçu do Sul, RS. Pelotas: Embrapa Clima Temperado, 2010. 28 p. (Embrapa Clima Temperado. Circular técnica, 107).

DELITTI, W. B. C. Ciclagem de nutrientes minerais em matas ciliares. In: SIMPÓSIO SOBRE MATA CILIAR, 1989, São Paulo. Anais... São Paulo: Fundação Cargil, Secretaria do Meio Ambiente, Instituto de Botânica, 1989. p. 88-98.

DELITTI, W. B. C. Estudos de ciclagem de nutrientes: instrumentos para a análise funcional de ecossistemas terrestres. Oecologia Brasiliensis, Rio de Janeiro, v. 1, p. 469-486, 1995.

FERREIRA, D. F. SISVAR: um programa para análises estatísticas e ensino de estatística. Revista Symposium, Lavras, v. 6, p. 36-41, 2008.

FIGUEIREDO FILHO, A.; MORAES, G. F.; SCHAAF, L. B.; FIGUEIREDO, D. J. Avaliação Estacional da deposição de serapilheira em uma Floresta Ombrófila Mista Localizada no Sul do Paraná. Ciência Florestal, Santa Maria, RS, n. 1, p. 11-18, 2003.
FORTES, O. F. Plano amostral para coleta de serapilheira na Floresta Ombrófila Mista no estado do Rio Grande do Sul. 2007. Tese (Doutorado em Engenharia Florestal) - Universidade Federal de Santa Maria, Santa Maria, RS.

JORDAN, C. F. Nutrient cycling in tropical forest ecosystems. New York: John Wiley \& Sons, 1985. 190 p.

KOLM, L.; POGGIANI, F. Ciclagem de nutrientes em povoamentos de Eucalyptus grandis submetidos a pratica de desbastes progressivos. Scientia Forestalis, Piracicaba, n. 63, p. 79-93, 2003.

KÖNIG, F. G.; SCHUMACHER, M.V.; BRUN, E. J.; SELING. I. Avaliação da sazonalidade da produção de serapilheira uma Floresta Estacional Decidual no Município de Santa Maria - RS. Revista Árvore, Viçosa, MG, n. 4, p. 429-335, 2002.

LOPES, M. I. S.; DOMINGOS, M.; STRUFFALDI-DE VUONO, Y.; Ciclagem de nutrientes minerais. In: SYSLVESTRE, L. S.; ROSA M. M. T. Manual metodológico para estudos botânicos na Mata Atlântica. Seropédica: EDUR, UFRRJ, 2002. p. 72-102.

MARTINS, S. V.; RODRIGUES, R. R. Produção de serapilheira em clareiras de uma floresta estacional semidecidual no município de Campinas, SP. Revista Brasileira de Botânica, São Paulo, v. 22, n. 3, p. 405-412, 1999.

MOREIRA, P. R.; SILVA, O. A. Produção de serapilheira em área reflorestada. Revista Árvore, Viçosa, MG, n. 1, p. 49-59, 2004.

MORELLATO, L.P.C. 1992. Sazonalidade e dinâmica de ecossistemas florestais na Serra do Japi. In História natural da Serra do Japi: ecologia e preservação de uma área florestal no Sudeste do Brasil. (L.P.C. Morellato, ed.)., Editora UNICAMP, Campinas, p.98-109.

OLSON, J. S. Energy storage and the balance of producers in ecological systems. Ecology, Tempe, v. 44, p. 322-331, 1963.

SCHLITTLER, F. H. M.; DE MARINIS, G.; CÉSAR, O. Decomposição da serapilheira produzida na floresta do Morro do Diabo (região do Pontal do Paranapanema, Estado de São Paulo). Naturalia, v. 18, p. 149-156, 1993.

SCHUMACHER, M. V.; BRUN, E. J.; HERNANDES, J. I.; KÖNIG, F. G. Produção de serapilheira em uma floresta de Araucaria angustifolia (Bertol.) Kuntze no município de Pinhal Grande-RS. Revista Árvore, Viçosa, MG, v. 28, n. 1, p. 29-37, 2004.

SHANKS, R.; OLSON, J. S. First year breakdown of leaf litter in Southern Appalachia Forest. Science, v. 134, p. 194-195, 1961.

VITAL, A. R. T.; GUERINI, I. A.; FRANKEN,W. K.; FONSECA, R. C. B. Produção de serapilheira e ciclagem de nutrientes de uma Floresta Estacional Semidecidual em Zona Ripária. Revista Árvore, Viçosa, MG, n. 6, p. 793-800, 2004.

WERNECK, M. S.; PEDRALLI, G.; GIESEKE, L. F. Produção de serapilheira em três trechos de uma floresta semidecídua com diferentes graus de perturbação na Estação Ecológica do Tripuí, Ouro Preto-MG. Revista Brasileira de Botânica, São Paulo, v. 24 n. 2, p. 195-198, 2001.

XU, X. N.; HIRATA, E. Forest floor mass and litterfall in Pinus luchuensis plantations with and without broad-leaved trees. Forest Ecology and Management, Amsterdam, v.157, p.165-173, 2002. 
